\title{
From isovists to visibility graphs: a methodology for the analysis of architectural space
}

\author{
Alasdair Turner \\ VR Centre for the Built Environment; e-mail: alasdair.turner@ucl.ac.uk \\ Maria Doxa \\ Bartlett School of Graduate Studies; e-mail: m.doxa@ucl.ac.uk \\ David O'Sullivan \\ Centre for Advanced Spatial Analysis; e-mail: david.osullivan@ucl.ac.uk \\ Alan Penn \\ VR Centre for the Built Environment; e-mail: a.penn@ucl.ac.uk \\ University College London, 1-19 Torrington Place, Gower Street, London WC1E 6BT, England \\ Received 15 November 1999; in revised form 19 February 2000
}

\begin{abstract}
An isovist, or viewshed, is the area in a spatial environment directly visible from a location within the space. Here we show how a set of isovists can be used to generate a graph of mutual visibility between locations. We demonstrate that this graph can also be constructed without reference to isovists and that we are in fact invoking the more general concept of a visibility graph. Using the visibility graph, we can extend both isovist and current graph-based analyses of architectural space to form a new methodology for the investigation of configurational relationships. The measurement of local and global characteristics of the graph, for each vertex or for the system as a whole, is of interest from an architectural perspective, allowing us to describe a configuration with reference to accessibility and visibility, to compare from location to location within a system, and to compare systems with different geometries. Finally we show that visibility graph properties may be closely related to manifestations of spatial perception, such as way-finding, movement, and space use.
\end{abstract}

\section{Introduction}

The concept of an isovist has had a long history in both architecture and geography, as well as mathematics. Tandy (1967) appears to have been the originator of the term 'isovist'. He presents isovists as a method of "taking away from the [architectural or landscape] site a permanent record of what would otherwise be dependent on either memory or upon an unwieldy number of annotated photographs" (page 9). The same idea has a similarly long history in the guise of the 'viewshed' in the field of landscape architecture and planning (Amidon and Elsner, 1968; Lynch, 1976) and in terms of 'intervisibility' in computer topographic models (Gallagher, 1972).

The appeal of the concept is that isovists are an intuitively attractive way of thinking about a spatial environment, because they provide a description of the space 'from inside', from the point of view of individuals, as they perceive it, interact with it, and move through it. As such, isovists have particular relevance to architectural analysis. Benedikt (1979) introduced a set of analytic measurements of isovist properties to be applied to achieve quantitative descriptions of spatial environments. Benedikt starts by considering the volume visible from a location and then simplifies this representation by taking a horizontal slice through the 'isovist polyhedron'. The resulting 'isovists' are always single polygons without holes, as shown in figure 1 (see over). Consequently, Benedikt considers geometric properties of isovists, such as area and perimeter. Thus he begins to quantify space, or what our perception of space might be, and the potential for its use. Benedikt notes that, in order to quantify a whole configuration, more than a single isovist is required and he suggests that the way in which we experience a space, and how we use it, is related to the interplay of isovists. This leads 


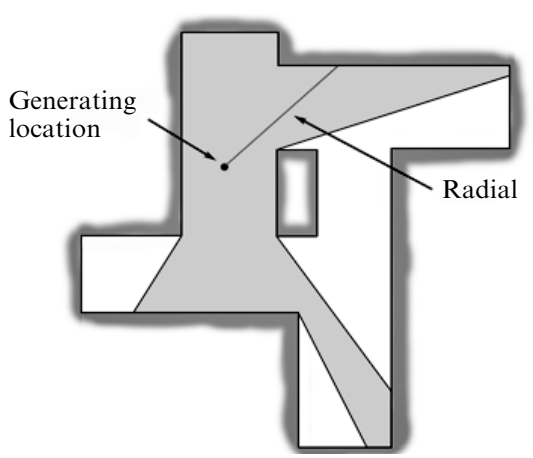

Figure 1. An isovist polygon, incorporating the visible area from a generating location (or convergence location of the optic rays).

him to formulate an 'isovist field' of his measurements. Isovist fields record a single isovist property for all locations in a configuration by using contours to plot the way those features vary through space. The packing of the contours shows how quickly the isovist property is changing and thus, Benedikt suggests, relates to both Gibson's (1979) conception of ecological visual perception and Giedion's (1971) classification of architectural types.

However, despite the elegance of Benedikt's isovist methodology, and its close relationship to theories of visual perception and spatial description, applications of the isovist in architectural analysis have been limited to a small number of studies. There appear to be two main reasons for this. First, the geometric formulation of isovist measures means that they index purely local properties of space, and the visual relationship between the current location and the whole spatial environment is missed-including the fact that the internal visual relationships between locations within the isovist are ignored. Second, Benedikt makes no propositions about how to interpret usefully the result of his isovist measures, although Benedikt and Burnham (1985) do show how perception of space is affected by various isovist attributes. In other words, there is little in the way of a theoretical framework to allow one to say how isovists relate to social or aesthetic matters. To overcome these limitations we introduce a broader methodology, one that embraces how visual characteristics at locations are related and one that has a potential 'social' interpretation. We draw on graph-based representations used in social theories of networks, primarily the space syntax theory of Hillier and Hanson (1984) and the small worlds analysis of Watts and Strogatz (1998), which leads us to use isovists to derive a visibility graph of the environment - the graph of mutually visible locations in a spatial layout. Through this representation we can obtain numerous measures of both local and global spatial properties that seem likely to relate to our perception of the built environment. By looking at these local and global properties, considering their meaning in terms of spatial description, and comparing them with actual usage-through movement and occupation of the environment that the graph represents - we hope to shed light on the effects of spatial structure on social function in architectural spaces.

\section{Related work}

Various authors have used Benedikt's formulation in architectural case studies. For example, Hanson (1994) uses isovists among other methods in order to investigate several well-known architects' houses. However, although Benedikt's work has had an impact on the way we think about space, there has been relatively little development of the isovist concept or related methodologies in the architectural literature. Davis and Benedikt (1979) make a more formal mathematical study of isovist generation and location, and Benedikt and Burnham (1985) study the perceptual impact of isovist 
properties. However, in the field of geoinformation science (GISci), the methodology has been explored more thoroughly under the moniker of viewshed analysis. Viewshed analysis concentrates on landscape, rather than urban and architectural issues (for a background to the technique, see Burrough, 1986, pages 39-56). Consequently, the area a viewshed covers is not necessarily continuous. For example, an observer may see over a ridge to a mountain in the distance, missing the intervening space. As for the extension of the methodology, there is a technical literature investigating the dependence of various viewshed characteristics on the accuracy of the underlying elevation data (for example, see Fisher, 1991; Huss and Pumar, 1997) and corresponding suggestions for the generation of 'fuzzy' viewsheds (Fisher, 1995). There also continue to be innovations in algorithms for generating viewsheds (Mills et al, 1992; Wang et al, 1996) and new suggestions for variants on the basic idea which determine the position of horizons, the vertical displacement of horizons from the viewpoint, and 'offset viewsheds' which take into account viewer height (Fisher, 1996). Of more relevance to the current work is viewshed analysis in archaeology (Wheatley, 1995), where the cumulative viewsheds over a region are used to determine the most visually prominent locations. The 'prominence' value generated is related to the distribution of different kinds of ancient monuments. More recent work looks set to extend this analysis considerably, by using differential measures of viewshed fields to measure more abstract concepts such as enclosure (Llobera, 1996). Llobera's work is similar in many respects to Benedikt's isovist fields. Indeed, much of the viewshed literature still concentrates on mapping the properties of single viewsheds as fields, not on the relationships between viewsheds. In work applying such structures to the classification of landscapes, Lee and Stucky (1998) have demonstrated the determination of routes with desirable visual characteristics. Their method finds the shortest path (according to the metric under consideration) through an implicit graph, in order to determine paths with particular characteristics, for example, the most scenic or most concealed route between two points.

Whereas in viewshed analysis graphs of visibility relationships are still implicit, architectural analysis has had a long history of graph-based analysis. Steadman (1973) demonstrates how graphs may be constructed of architectural arrangements, considering relationships between architectural units (such as rooms or corridors), although the original concept dates back further (for example, see March and Steadman, 1971; Ore, 1963). Krüger (1979) shows how similar graphs of the relationships between urban units may be constructed. In contrast to forming edges between structural units, Hillier and Hanson (1984) introduce visibility relationships into graph analysis of buildings and urban systems. They construct the set of axial lines for a system, which are the fewest longest lines of sight and access in the system which traverse all the convex spaces within that system (the convex spaces being a nearminimal set of nonoverlapping convex polygons covering the space $\left.{ }^{(1)}\right)$. The axial lines thus form a set of intersecting lines which represent all nontrivial rings of circulation in a system. By graphing the structure of axial lines (where each axial line is a vertex in the system, and each intersection a node) Hillier and Hanson provide a description of how the system can be traversed in terms of lines of sight, because we can say how many changes of direction are required to reach any space from any other space in the system. The methodology may be extended by introducing new graph representations capable of being automated, such as 'all-line' axial maps, in which all the lines that

(1) Constructing a unique minimal set of convex polygons is not possible in most cases, or at any rate not calculable in polynomial time (see deBerg et al, 1997, pages 45-61), but Hillier and Hanson show that, in practice, it is simple to decide on a sensible spatial breakup for a given morphology by choosing the 'shortest and fattest' convex polygons. 
form a tangent between any pair of mutually visible vertices are drawn (Hillier and Penn, 1992). This representation produces a more densely packed graph, with more information about the relation of lines of sight in a system. Despite their general applicability, the ability of these maps to represent and quantify spatial configurations still has one major drawback: each line or convex region is represented by a node in the graph, and so only a single graph measure can be defined for points along the whole length of the line, or all points within the convex region. Hillier et al (1995) combine line-of-sight measures and tessellation of areas to resolve this issue. However, as isovists can be drawn at any location in space, a graph of lines-of-sight connections may be constructed easily, at any required degree of spatial resolution, by using the visual relationships between isovists. De Floriani et al (1994) propose such a form of graph, a visibility graph, which is identical in structure to that which we propose here, albeit to solve the problem of transmitter placement rather than analysis of the underlying landscape properties and thus their approach is very different.

\section{Constructing an isovist graph}

Constructing an isovist graph of a spatial environment involves two distinct sets of interrelated decisions. First, we must select an appropriate set of isovists (in fact an appropriate set of generating locations, according to some criterion) to form the vertices of the graph. Second, given a particular set of isovists, we must determine which relations between them are significant, or are of interest, to form edges in the graph. These steps are both theory laden and must be driven at least in part by pragmatic considerations - the size of the graphs which will be produced, and so on. Here we explain the decisions we have made. The theoretical implications of these decisions will become clearer in the presentation of the various graph-based measures we have used. For now we acknowledge that there is no compelling way of choosing one particular approach over another and we discuss some avenues for further research later in the paper.

Ideally we would like to select some set of isovists that 'fully describes' the spatial system. In practice we must compromise and try to select a set of generating locations that provides an acceptable 'near-full' description of the space. Following Benedikt, we assume that isovists can be meaningfully generated throughout a space and furthermore that it is useful to examine isovist properties (whether local or relational) throughout a system. Thus the most obvious approach is to generate isovists throughout a spatial system at some regularly spaced interval. This implies that the generating locations will be at points defined by some sort of grid or regular lattice. The appropriate grid resolution must then be determined. If analysis is to relate to human perception of an environment, then the resolution of this grid must be fine enough to capture meaningful features of the environment. On the other hand, if we wish to consider human usage of an environment, a lower resolution may be used (for example, mapping only space that is humanly accessible). We have adopted the pragmatic approach of using a 'human-scale' grid spacing of around one metre. This seems reasonable given that our purpose is to understand spatial environments designed for human occupation as they are used and perceived by individuals. However, ultimately, the resolution of the analyses we present here is limited only by available computing power.

Of course, a method approximating the underlying space such as this quickly produces very large sets of generating locations. To remedy this we might try to find a minimal covering set of isovists for the space. For example, we might start by choosing the most 'strategic' location in the environment, then continue by selecting additional locations which maximise the area viewable from the set as each location is 
added. However, although finding a sufficient set by this method is possible, it is not guaranteed to be a minimal covering set (Davis and Benedikt, 1979). In addition, for our purposes, a graph analysis of any such minimal set is likely to provide only relatively obvious information about the environment, because locations at the end of long corridors or at prominent street junctions will tend to be favoured. However, note that this issue is central to problems considered in GISci, such as line-of-sight communications.

Once we have selected a set of generating locations, which relationships between different isovists in an environment should be included in an isovist graph? If we consider the isovists to be polygons, the most obvious relationship to consider occurs where two isovist polygons intersect with one another. Arguably, a stronger relationship between two isovists exists where they intersect and their generating locations are mutually visible. We refer to this as a first-order relationship. In order to determine this relationship we need not invoke isovists at all: we can simply make a graph with physical locations as vertices, and form edge connections between pairs of locations if they are mutually visible. This is a visibility graph of the system ${ }^{(2)}$. As, by definition, an isovist from a given generating location contains all the locations visible from it, the visibility graph is identical to our putative first-order isovist graph. An 'isovist intersection graph' is closely related: it can be formed by taking a visibility 'step' from one isovist-generating location to an intervening location, and then a 'step' onto the next isovist-generating location. Hence we refer to this as a second-order visibility relationship (see figure 2). It is apparent from this description that a second-order visibility or isovist intersection graph is a 'flattened' form of the (first-order) visibility graph, where the set of edges is the union of all the one-step and all the two-step edges. Thus the first-order visibility graph contains all the information necessary to form the second-order graph and therefore we will concentrate on the analysis of first-order

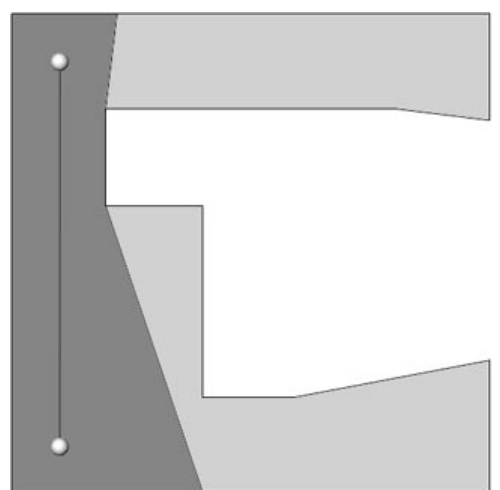

(a)

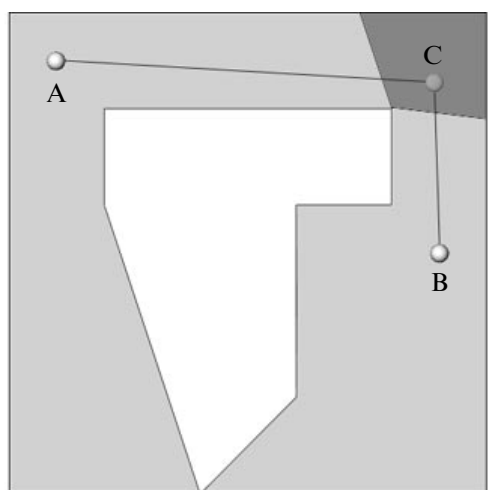

(b)

Figure 2. (a) First-order and (b) second-order visibility relationships between isovists. The second-order graph is just a 'flattened' first-order graph-A is linked directly to B, rather than through $\mathrm{C}$ as it would be in the first-order case.

(2) The term visibility graph was introduced to landscape analysis by De Floriani et al (1994) and it is also prevalent in computational geometry and artificial intelligence (see deBerg et al, 1997, pages $305-315$ ). Both of these forms of visibility graph are more sparsely connected than our own, as they include only key locations in the environment (in landscapes, points selected from a triangulated irregular network (TIN); in computational geometry, corners of two-dimensional polygons). However, the architectural visibility graph also uses selected locations as vertices and a mutual visibility relationship to form edges, and thus all the graphs are of identical form. 


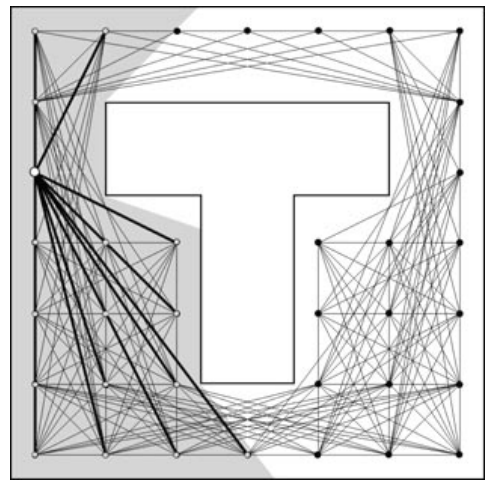

Figure 3. An example of a first-order visibility graph, showing the pattern of connections for a simple configuration.

visibility graphs in this study. Figure 3 shows an example graph made from thirty-six point locations.

Note that, although we have discussed a graph in terms of visibility, and therefore implicitly at eye level, the visibility graph can be formed by taking an isovist at any height above the floor. As Hanson (1999, page 54) writes "In moving around in buildings, people orientate themselves by reference to what they can see and where they can go. [In addition,] in looking at the visual and volumetric qualities of architecture, we need not be constrained by the pragmatics of everyday space use and movement. Indeed, we should not be, since architectural speculation almost invariably brings into play the relationship between visibility (what you can see) and permeability (where you can go)." Thus it seems sensible to extend our analysis to both visibility and permeability graphs of systems. To be clear, a 'permeability graph' is the special case of a visibility graph constructed at floor level. We will see how the permeability graph varies according to whether furniture and other obstacles to accessibility are included in the analysis of a system.

In mathematical terms, a graph consists of two sets: the set of the vertices in the graph, labelled $V$, and the set of edge connections joining pairs of vertices, labelled $E$ (for an introduction to graph theory, see, for example, Wilson, 1996). This information is summarised by writing the graph as the pair of these sets: $G(V, E)$. In the case of a visibility graph, the vertices represent the set of generating locations to be considered:

$$
V=\left\{v_{1}, v_{2}, v_{3}, \ldots, v_{n}\right\} .
$$

The edges are pairs of mutually visible points. We will denote the edge joining $v_{1}$ and $v_{2}$, that is $\left\{v_{1}, v_{2}\right\}$, as $e_{12}$. Thus the edge set will be of the form

$$
E=\left\{e_{12}, e_{23}, \ldots, e_{i j}\right\}, \quad \text { where } e_{i j} \Leftrightarrow e_{j i} .
$$

In a representation on a two-dimensional plane, the graph edges are undirected (that is, if $v_{1}$ can see $v_{2}$, then $v_{2}$ can see $v_{1}$ ). However, in other representations visibility is not necessarily mutual - for example, viewshed analyses often consider viewer height. In addition, even ignoring viewer height, we may want to construct an 'accessibility' graph, differentiating between, for example, escalators or entrances and exits. In such cases, edges in the graph may be directed. Many possible measures of an undirected graph are also applicable to a directed graph, including those we illustrate in this paper, so that the generality of the method presented is unaffected. 


\section{Analysing the graph}

Having derived a visibility graph for a spatial environment we can analyse it by making use of some of the many measures developed for investigating graph properties across a number of disciplines [Wilson and Beineke (1979) give an idea of the range of measures available]. We focus on three measures of graph structural properties. These are the local properties neighbourhood size and clustering coefficient, and the global property mean shortest path length. The clustering coefficient and mean shortest path length have previously been used together to characterise graph systems as a whole (see Watts and Strogatz, 1998) and we discuss how this characterisation might be applied to visibility graphs of environments in further work. However, for the current study, we consider each measure from the point of view of each vertex in the graph and we examine the pattern of their distribution across systems. This is readily done by mapping the values of measures at each generating location by using some colour scale. In the sections that follow, we describe the measures in detail, discuss their likely usefulness and implications, and present some cases based on the analysis of various relatively simple examples.

\section{Neighbourhood size}

The neighbourhood of a vertex is the set of vertices immediately connected through an edge. Expressed in terms of graph notation, the neighbourhood $N_{i}$ of a location $v_{i}$ is the set of directly visible vertices:

$$
N_{i}=\left\{v_{j}: e_{i j} \in E\right\} .
$$

Now if the set of generating locations covers the entire space (at some uniform resolution, so that for our purposes it fully describes the space), then this set can be thought of as equivalent to the isovist itself. Hence there is a one-to-one correspondence between the neighbourhood of a vertex in a visibility graph and the isovist from the location represented by that vertex. (Note that the neighbourhood of a location does not include itself. Technically we should write that $N_{i} \cup v_{i}$, not $N_{i}$, corresponds to an isovist.) Thus we can attach a meaningful spatial description to the neighbourhood size: it is linearly related to the isovist area, that is the area $A_{i}$ of the isovist at location $v_{i}$ is directly proportional to the neighbourhood size $k_{i}$ of the vertex $v_{i}$ :

$$
A_{i} \propto\left(k_{i}+1\right), \quad \text { where } k_{i}=\left|N_{i}\right| .
$$

(We assume adequate coverage of generating locations throughout the system. In figure 3 the coverage is not sufficient and the isovist area no longer corresponds perfectly to the neighbourhood size.)

We can plot the values of neighbourhood size for all the physical locations represented by vertices in the graph. This kind of plot has direct relevance to Benedikt's description of space by isovist fields - a plot of the isovist field of the area property draws contours of equal viewable area across a space. In figure 4 (see over) we show the isovist field of isovist area for a simple spatial configuration, and beside it the neighbourhood size plotted by using a scale from black (minimum area) to white (maximum area). Note that the isovist field lines are continuous, whereas the discrete generating locations used to construct the visibility graph can be seen in the second diagram.

\section{Clustering coefficient}

The clustering coefficient is defined as the number of edges between all the vertices in the neighbourhood of the generating vertex (that is, the number of lines of sight between all the locations forming the isovist) divided by the total number of possible connections with that neighbourhood size. In isovist terms this is equivalent to finding 


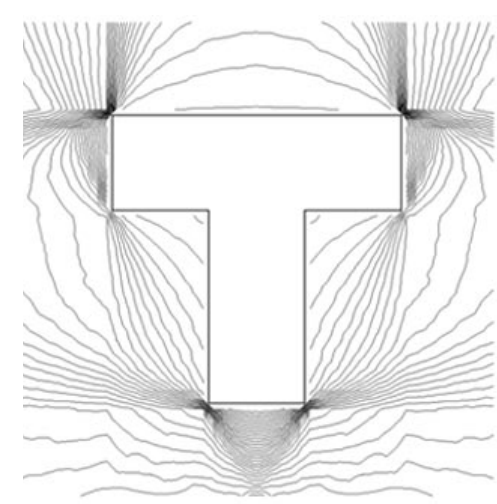

(a)

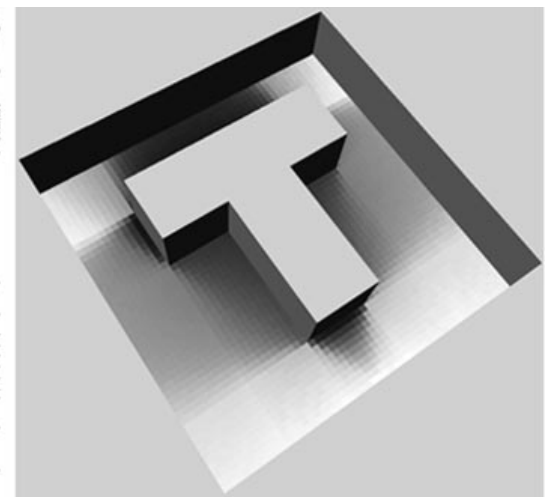

(b)

Figure 4. (a) An isovist field of isovist area, and (b) a plot of neighbourhood size values.

the mean area of intersection between the generating isovist and all the isovists visible from it, as a proportion of the area of the generating isovist. In set terms, the clustering coefficient $C_{i}$ for the neighbourhood $N_{i}$ of location $v_{i}$ is

$$
C_{i}=\frac{\left|\left\{e_{j k}: v_{j}, v_{k} \in N_{i} \wedge e_{j k} \in E\right\}\right|}{k_{i}\left(k_{i}-1\right)},
$$

where $k_{i}$ is the neighbourhood size.

At first sight this measure relates to the convexity (or conversely the 'spikiness') of the isovist at the generating location $v_{i}$. If the isovist being considered is almost a convex polygon, then almost all the point locations within the neighbourhood will be able to see each other, and hence $C_{i}$ will tend to one. If, on the other hand, the isovist is very 'spiky' (not at all convex) then many points within the isovist will not be visible from each other, and $C_{i}$ will tend to zero. Figure 5(a) shows $C_{i}$ mapped for a simple spatial configuration, for a graph with 2260 vertices.

Further consideration reveals that the clustering coefficient gives a measure of the proportion of intervisible space within the visibility neighbourhood of a point. It indicates how much of an observer's visual field will be retained or lost as he or she moves away from that point. If the neighbourhood of a point approximates a convex

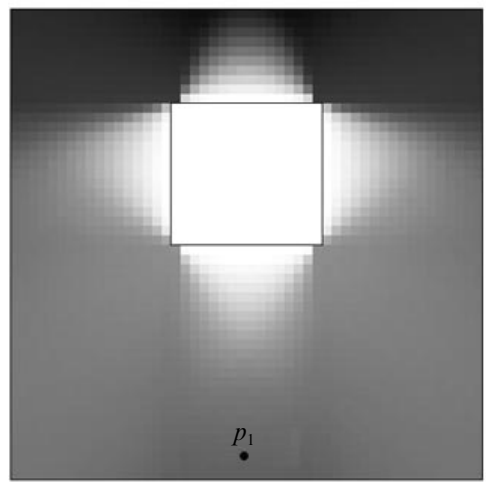

(a)

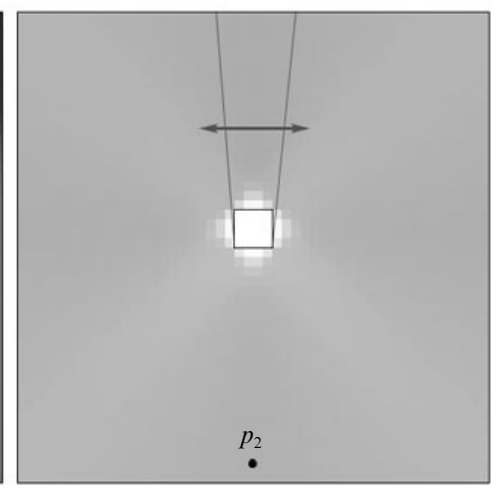

(b)

Figure 5. (a) Clustering coefficient values for a simple configuration. (b) The clustering coefficient is increased when many points within the isovist are mutually visible, regardless of the convexity of the geometric isovist polygon. 
polygon, then the clustering coefficient is high and moving from that location in any direction will not cause any great loss of visual information. However, at a junction with multidirectional visual fields, $C_{i}$ will be low as moving from that location will involve loss of part of the currently visible area. Because movement in some sense involves making decisions about which parts of one's current visual information to leave behind, the clustering coefficient is potentially related to the decisionmaking process in way-finding and navigation and certainly marks out key decision points within complex configurations. Further, if we regard vertices in the graph as potentially occupied by people, $C_{i}$ values indicate the potential for perceivable copresence in a space and therefore the potential to form groups or to interact ${ }^{(3)}$. In a closed convex area there is some potential for interaction, whereas in a junction there are numerous, but different, opportunities to form intervisible links. This seems likely to prove a useful property in studying the perception of spaces and may also be useful in behavioural studies. For example, Benedikt and Burnham (1985) show that perception of the size of a space is affected by the variance of the radials of an isovist and the perimeter (both indicators of convexity). In addition, de Arruda Campos (1997) finds good correlation with the number of axial lines intersecting at an urban space (implying a multidirectional visual field, that is, low $C_{i}$ ) and the number of people making informal use of the square, and Conroy (2000) notes correspondence between 'junctions' and places where people pause on journeys.

We use the clustering coefficient to analyse two houses as examples: Alvar Aalto's Villa Mairea and Mies van der Rohe's Farnsworth House (for background information on the houses, see Curtis, 1996). Figure 6 shows the pattern of $C_{i}$ values produced for the interior spaces of the Villa Mairea. The visibility graph is formed by taking a $1.0 \mathrm{~m}$ grid on a section of the plan at eye level on each floor, then linking vertices on the staircases across the two levels to produce a single graph. Afterwards, $C_{i}$ values are calculated at each location. The figure shows that the most private spaces, such as bedrooms and study rooms, are highly clustered whereas social spaces, such as the living rooms on each floor and ground-floor sitting rooms offer multidirectional fields of view and therefore low $C_{i}$, values, hinting at - without intruding on-the range of the more private spaces.

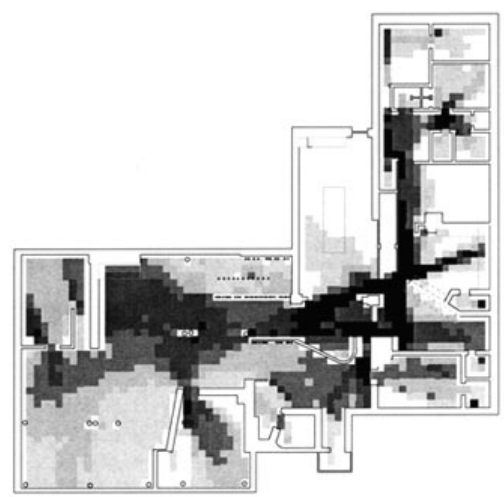

(a)

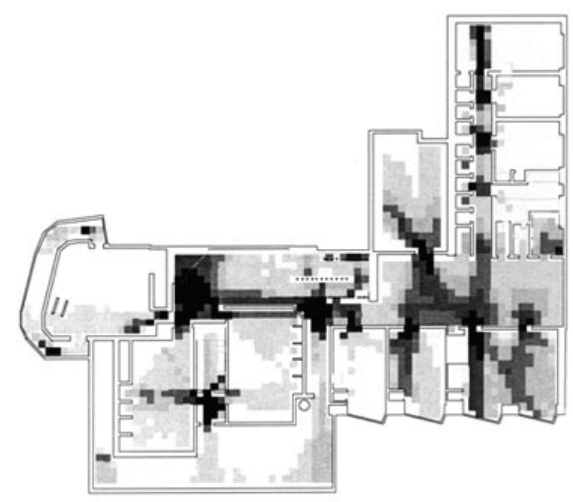

(b)

Figure 6. The clustering coefficient measured for Aalto's Villa Mairea. The two floors have been linked via the stairwells.

(3) We realise that any population of a space will interfere with visual fields, and thus $C_{i}$. However, the presence of individuals is transient and, as lines of sight are restored with movement (of both the observer and the observed), we believe appreciation of the built form as a forum for interaction remains possible. 
The results of clustering coefficient analysis are related to the endpoint partitioning proposed by Peponis et al (1997). The spaces defined by endpoint partitions, e-spaces, are mathematically well defined and lead to a unique spatial description. They define 'informationally stable' units with respect to form, by considering the visibility of discontinuities of shape, such as corners and edges of wall surfaces. Being dependent on discontinuities, they are affected by the complexity of the plan and the degree of detail in the representations of shapes. As a spatial description, e-spaces present a different approach to visibility graphs, primarily because they are derived by relating space to built form, rather than derived from the space as it might be experienced. However, e-spaces and $C_{i}$ values both provide an indication of the internal cohesion of the overall space at any point within that space. Peponis et al presented results of their analysis for a simplified plan of Farnsworth House, and their diagram (page 772) shows strictly delineated areas of informational stability. In contrast, analysis of a plan of Farnsworth House with $C_{i}$ values results in a gradual partitioning of space, where information is seen to vary continuously across the space and within e-spaces [figure 7(a)]. Although there is 'informational stability' with respect to vertical surfaces there are clear variations in the internal relational properties of the isovists within these spaces. Considering the $C_{i}$ values in more detail, there is a maximum at locations closer to the central element, which is more self-contained, with almost convex visual fields. The lowest values occur further away where isovists are multidirectional (and Hillier and Hanson's convex partitioning schema is difficult to apply). In these locations, the placement of the furniture by the users reorganises the spatial layout by relating accessibility and/or permeability to the lived use of space-as shown in figure 7(b), which demonstrates clustering coefficient permeability analysis of Farnsworth House with its furniture.

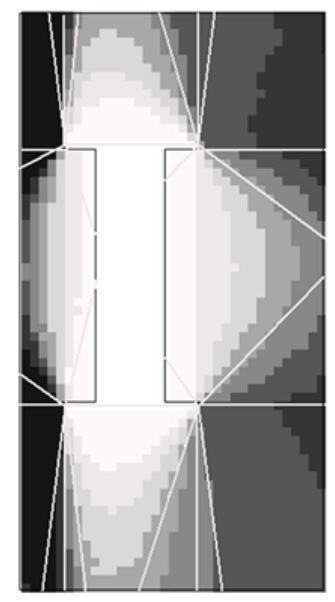

(a)

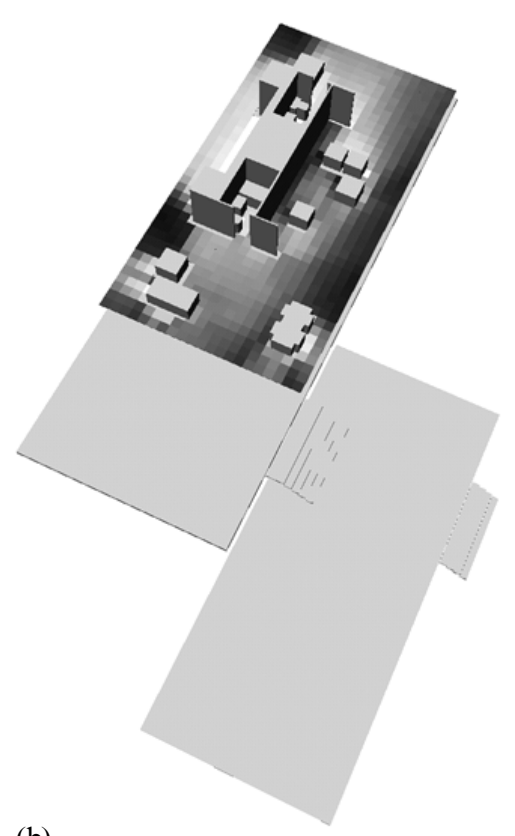

(b)

Figure 7. (a) Clustering coefficient values for a simplified Mies van der Rohe's Farnsworth House, analysed for visibility with the e-partitions of Peponis et al overlaid; and (b) clustering coefficient values for the building furnished and analysed for permeability. 
Note that the clustering coefficient might be considered similar to a convexity measure of an isovist polygon, such as some combination of Benedikt's measures of variance of the radials $M_{2}$ and perimeter $P$. However, the $C_{i}$ measure is in fact more subtle than this, precisely because the geometry of the isovist polygon has been discarded. Any pair of mutually visible locations within the isovist area contribute to the overall $C_{i}$ value; thus any system with 'visibility loops' will display higher $C_{i}$ values than those without. For example, both figures $5(\mathrm{a})$ and $5(\mathrm{~b})$ plot $C_{i}$ values by using the same scale. The isovist polygon at $p_{1}$ is more convex (in terms of $M_{2}$ and $P$ ) than that at $p_{2}$, yet the value of $C_{i}$ at $p_{2}$ is higher. Hence the clustering coefficient is not strictly a convexity measure, but perhaps more related to how 'self-contained' the information in a particular isovist is. Thus, because $C_{i}$ is not just a measure of geometric 'spikiness', but also a measure of how much objects of varying sizes disrupt the space, it may improve our understanding of how a space is perceived. For example, although adding a pillar to a space will increase the spikiness of an isovist dramatically, people will not usually be fooled by such an addition [figure 5(b)]. Through movement they will still be able to perceive the space as a whole, and this is reflected in the clustering coefficient value.

The sensitivity of clustering coefficient analysis to the size of object in the environment would have a significant impact if we were to consider not just the internal relationships in Farnsworth House, but also its relationship to the surrounding environment. Further, the analysis we have made has not considered the built fabric, or the relation of the interior to the building's context. So far, we have taken no account of the fact that the external envelope of Farnsworth House is made entirely of glass, and this raises interesting questions with respect to visibility relations between 'inside' and 'outside'. We might consider the surrounding space (the stepped terraces and the landscape) and include visibility connections through the glass walls. As one approaches the house, it features as an object in the landscape and, on looking through the glass external wall, one finds that the core becomes an object within an object. At a distance, the clustering coefficient will remain relatively high, because the connections between isovists on either side of the house are realised in a loop around it [figure 8(a)]. As we move towards the entrance, through the terraces, the space becomes gradually more clustered and only the central core inside the building, visible through the glass, features as an object [figure 8(b)]. As we enter, the clustering coefficient increases

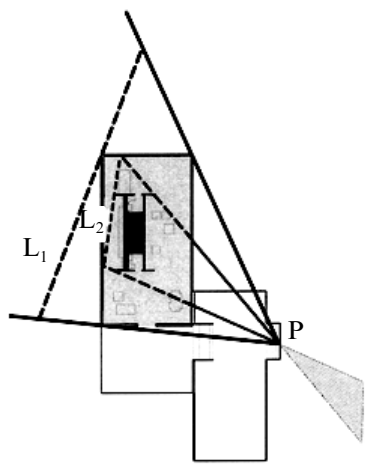

(a)

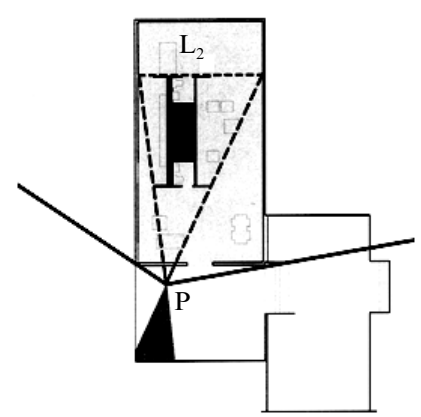

(b)

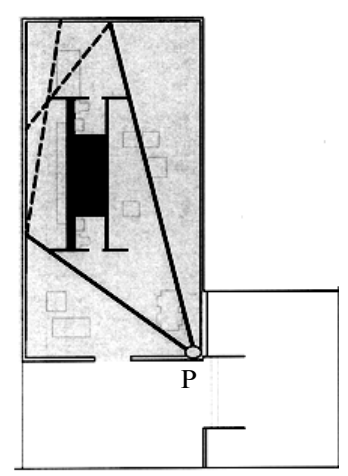

(c)

Figure 8. Direct connections between points on either side of an isovist from P, completing loops behind the Farnsworth House $\left(\mathrm{L}_{1}\right)$ and (assuming 'visibility' into but not out of the glass envelope) its central core element $\left(\mathrm{L}_{2}\right)$. In (a) both loops are completed, in (b) loop $\mathrm{L}_{2}$ cannot be completed owing to obstructions in the surrounding environment, and in (c) neither loop is completed. 
further towards the core element, which now becomes an architectural element articulating the interior space around it. In fact, if once inside we consider the walls as containing our 'visibility' (or at least our sense of enclosure), then there is no location within the house where the points of an isovist on either side of the core element connect in a loop behind it [figure 8(c)]. In this sense the clustering measure may capture the transition between architecture considered as a disposition of objects and architecture as a space-defining configuration, a transition which is dependent on the relative location of the viewer.

\section{Mean shortest path length}

The shortest path between two vertices in a graph is the least number of edges that need to be traversed to get from one vertex to the other. The mean shortest path length for a vertex is simply the average of the shortest path lengths from that vertex to every other vertex in the system, and so represents an average of the number of turns (plus one) required for any journey within the system. Formally defined, a path from $v_{i}$ to $v_{j}$ is a sequence of unique intervening vertices between $v_{i}$ and $v_{j}\left(v_{i}, \ldots, v_{n}, \ldots, v_{j}\right)$, such that consecutive vertices in the sequence are joined by an edge in the graph, that is, $e_{n, n+1}$ is a member of $E$. The length of a path is the number of edges it traverses, and the distance $d_{i j}$ between $v_{i}$ and $v_{j}$ is the length of the shortest available path between them. Thus the mean shortest path length $\bar{L}_{i}$ for a location $v_{i}$ in terms of graph notation is:

$$
\bar{L}_{i}=\frac{1}{|V|} \sum_{j}^{v_{j} \in V} d_{i j} .
$$

Figure 9(a) shows the mean shortest path length mapped for a simple spatial configuration, for a graph with 2000 vertices. The lower $\bar{L}_{i}$ values are coloured white in the figure, whereas higher $\bar{L}_{i}$ values are black.

Note that the measurement of mean shortest path length has direct parallels with Hillier and Hanson's approach. Hillier and Hanson quantify the visual accessibility of spaces (through the number of turns connecting those spaces), whereas we quantify the visual accessibility of every location in the spatial system (through the number of turns plus one). Hence our analysis extends the Hillier and Hanson method to (near) continuous space and enables the resulting locations within a space to be mapped across that space. Indeed, because this more direct representation quantifies the visual

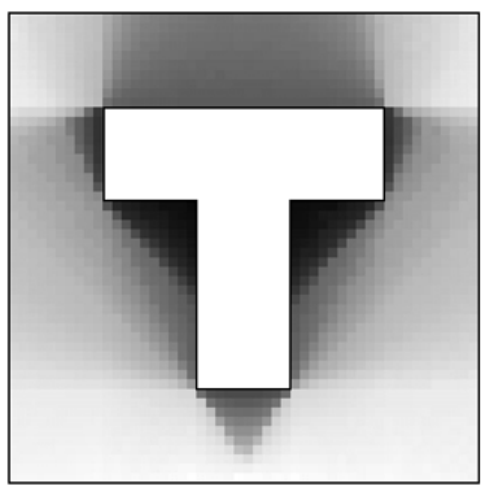

(a)

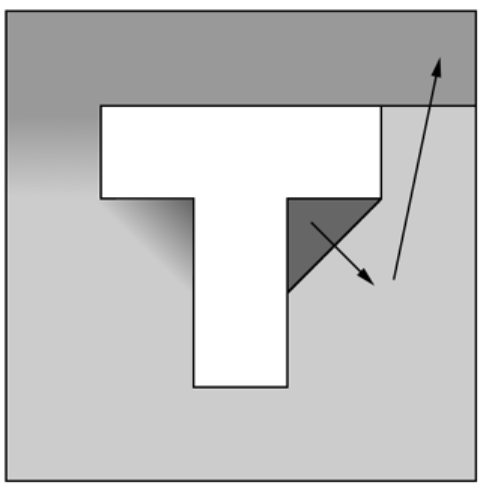

(b)

Figure 9. (a) Mean values of the shortest path length for a simple configuration. (b) The area under the T-crossbar has higher $\bar{L}_{i}$ values because of a two-step relationship with the area above the crossbar. 
accessibility of every location in the spatial system, it has a significant advantage over axial and convex configurational analysis, which, for example, is unable to identify variation across 'open-plan' layouts.

As the mean shortest path length measures configuration by considering locations with respect to each other location in the system, global relationships between locations in the system become apparent. For example, the points located below the T-crossbar in figure 9(a) form distinctly coloured triangular regions according to their $\bar{L}_{i}$ values. This is due to the second-order effect of spaces two steps away. In this case, because they cannot 'see' the top spaces of the configuration, an individual occupying that location would not know that there might be a place 'around the corner'. If an individual leaves one of these triangular zones, then they are entering a zone where, one step away, they can see a larger area, as shown in figure 9(b). Thus the pattern of $\bar{L}_{i}$ values classifies locations according to global configurational properties. As in the case of the triangular regions of the T-shape example above, this kind of partitioning does not show up in any obvious first-order partition, including such detailed descriptions as those of endpoint partitions by Peponis et al. This suggests that by using the visibility graph technique we obtain an alternative spatial description to those previously available by partitioning the space in terms either of local geometric properties of visual fields as Benedikt does, or of formal discontinuities and adjacency properties as Peponis et al do.

As a built example, we consider the configurational characteristics of the spaces in Mies van der Rohe's Barcelona Pavilion. The pavilion was built in 1929 for the German participation in the Barcelona exhibition and demolished afterwards. It was retrieved through photographs and plans, to be rebuilt in 1986 (for a detailed discussion, see Futagawa and Neumeyer, 1995). Benedikt uses the plans of this building to investigate isovist - field contour maps, demonstrating that the area of the visual field changes continuously with movement, as surfaces disappear and others come into view. We construct both a permeability graph as well as the visibility graph [compare figures $10(\mathrm{~b})$ and $10(\mathrm{c})$ ]. To create these figures we used a $0.5 \mathrm{~m}$ grid of vertices covering all accessible areas in the pavilion, forming two different graphs taken at eye height and floor height. In the visibility case, for example, edges are formed between points visible across the pool, or on opposite sides of a piece of

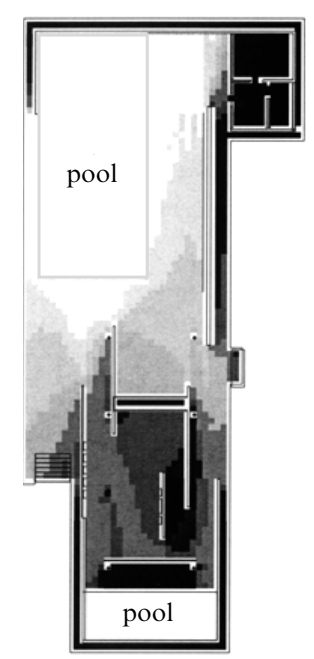

(a)

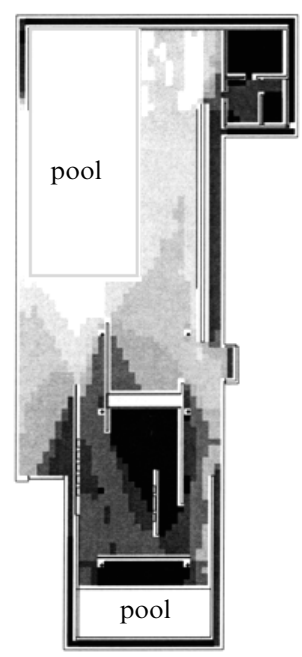

(b)

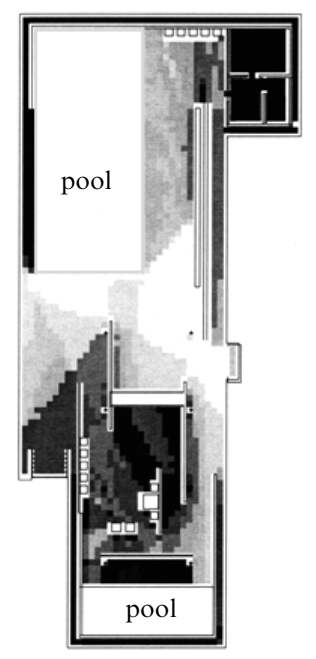

(c)

Figure 10. Mies van der Rohe's Barcelona Pavilion showing (a) neighbourhood size, (b) visibility mean shortest path length analysis, and (c) accessibility mean shortest path length analysis. 
furniture, whereas no connection is made in the permeability graph. In both cases, path length analysis produces a pattern that resembles that of neighbourhood size, or area of visual fields, and therefore is similar to Benedikt's area measurement, owing to the fact that this is a small or shallow system where the architect introduces partitions to structure the space without disturbing too much its continuity-compare the $k_{i}$ values in figure 10(a) with $\bar{L}_{i}$ values shown in figures $10(\mathrm{~b})$ and $10(\mathrm{c})$. However, path length analysis still enables us to look at the relationship between specific locations in the space and the configuration as a whole. The main difference in the configurational patterns generated results from the way the large pool is analysed. The comparison shows how the space has been manipulated by the architect to arrange views across the pool and the movement around it. Thus, in the visibility model, $\bar{L}_{i}$ is maximised at the edges of the system around the pool. When analysed in terms of accessibility, however, the core $\bar{L}_{i}$ values are shifted to the central space that links the two locations with the richest accessibility fields and spreads towards the far side offering views to the smaller pool and statue.

In larger and more complex systems than the Barcelona Pavilion, the local (such as $k_{i}$ ) and global (such as $\bar{L}_{i}$ ) characteristics vary considerably from each other. As an example of a more involved system we analyse the Tate Gallery on Millbank in London, in which there are many rooms consisting of both gallery space and major movement routes through the building. Figure 11(a) shows neighbourhood size and figure 11(b) shows the pattern of $\bar{L}_{i}$ values for the main level of the Tate Gallery, and the difference between the local and the global spatial measure is very obvious, now that many changes of direction are required for full exploration of the space.

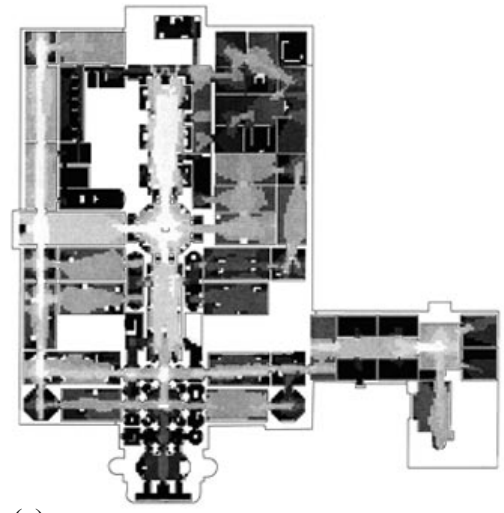

(a)

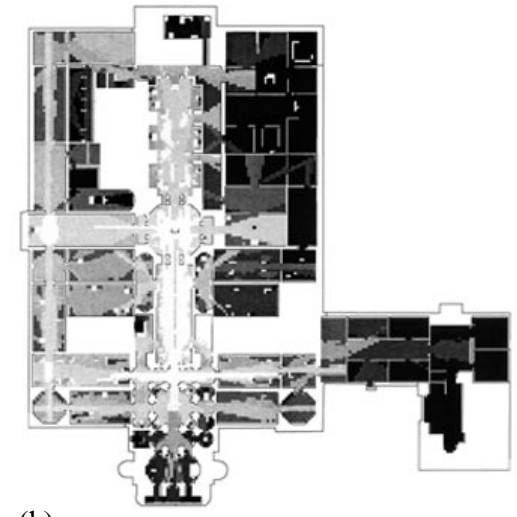

(b)

Figure 11. Visibility graph analysis of the Tate Gallery showing (a) the neighbourhood size, and (b) the pattern of mean shortest path length in the publicly accessible first-floor spaces.

However, we need not constrain mean shortest path length analysis to a qualitative discussion of spatial description. In a study of the Tate Gallery, Hillier et al (1996) demonstrate that there is a correlation between 'pesh' analysis (an $\bar{L}_{i}$-type measure for spaces within a configuration; see Hillier et al, 1995) and the number of people moving between predefined areas in the building. This indicates that comparing the $\bar{L}_{i}$ values across the visibility graph with people movement patterns may be fruitful. For example, figure 12(a) shows the movement patterns over the first ten minutes of a visit to the Tate Gallery. Comparing this with $\bar{L}_{i}$ values shown in figure 11(b), there appears to be a qualitative correspondence between the two. Correspondence can also be observed when we compare $\bar{L}_{i}$ values against average room occupancies during the day. Figure 12(b) shows the occupancy level per room, with data from the Hillier et al (1996) study 


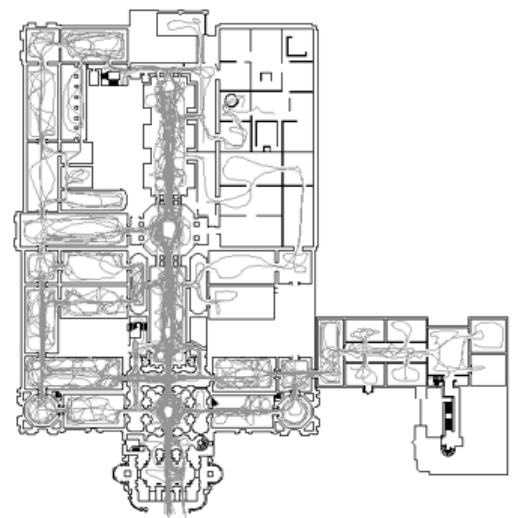

(a)

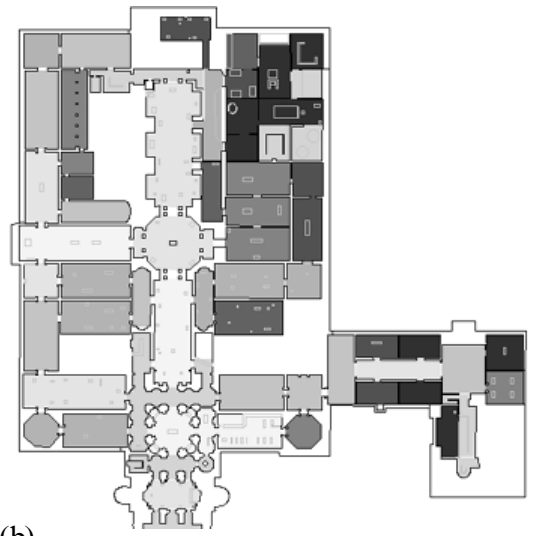

(b)

Figure 12. (a) First ten-minute movement traces of people through the gallery (Hillier et al, 1996, page 20), and (b) the average observed occupancy levels of rooms during the day.

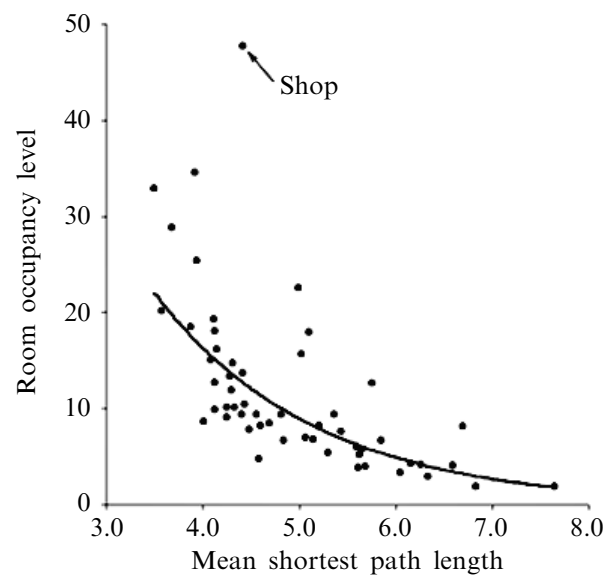

Figure 13. A scatter plot showing the room occupancy level (average number of people at any instant) against the mean shortest path length. [An exponential regression line $\left(R^{2}=0.63\right)$ is shown.]

of the Tate Gallery, where the spaces were observed five times in a day and averaged across the day. The correspondence is demonstrated more clearly by inspection of a scatter plot of observed room occupancy against $\bar{L}_{i}$ (see figure 13). In order to obtain the plot shown, the $\bar{L}_{i}$ values of the locations within each space have been averaged to give a single value per room ${ }^{(4)}$. A best-fit line through the data gives a reasonable exponential correlation between the two variables and it is noticeable that the only significant outlier found is the Tate Gallery shop, which is the only room that is not used as gallery space. Although a correspondence of this sort might be expected in view of the fact that we are mapping a measure of centrality, it is worth demonstrating that this is not a necessary result. Figure 14 (see over) shows a Euclidean version of $\bar{L}_{i}$ analysis (which, for each location, measures the mean Euclidean distance to all other locations in the space, on the same grid as before), applied as naïvely as $\bar{L}_{i}$ visibility

(4) Note that the $y$ axis shows the average occupancy in each room, not the average occupancy density. The fact that our measure still correlates might be expected, as $k_{i}$ (which, within a room, is roughly proportional to the area of the room) has the most significant effect on $\bar{L}_{i}$. 


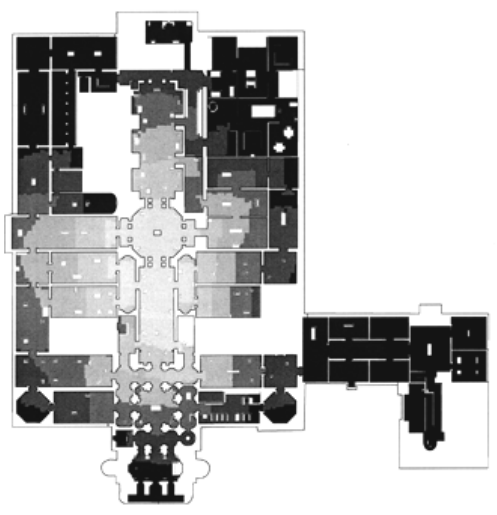

Figure 14. Mean Euclidean shortest path lengths for the Tate Gallery (average distance from each grid location to all other grid locations in the system).

graph analysis, and the result shows no obvious correspondence to either the first ten-minute movement or the average occupancy of the rooms.

We hope that in the future we may be able to relate correspondences such as those found in the Tate to some human cognitive model. Benedikt cites Gibson's (1979) model of visual perception as one of the major reasons for constructing contours in isovist fields, reasoning that movement decisions should be made at times of rapid change in isovist area values. Similarly we can propose tentative hypotheses. For example, if individuals are random agents in the environment, moving towards open space, then they would tend to coalesce in areas of high visual accessibility. As the agents' general movement is directed along lines of sight, more lines of sight meeting at a location would lead to a higher congregation of individuals at that location (a result mirrored by de Arruda Campos's findings). Although this is obviously a gross simplification of any actual interaction of individuals with a spatial system, it shows a clear way in which simple predictive models can be made and tested with the visibility graph method.

\section{Further work}

Although the characterisation of space in this way is interesting, much work on the application of the analysis is still required. Most importantly we need to develop a more systematic method of selecting and generating point locations. At the very least, the effect of the generating point locations on graph measures must be researched. The grids we currently use are dependent mainly on the power of the computers we use to analyse them. Nevertheless, however powerful the computer, we can never achieve 'perfect' resolution to represent the spatial environment. Necessarily we must compromise on the graph structure we choose and it would be preferable to have a firmer theoretical basis for this choice. According to van Fraassen's scientific empiricism (1980, pages 76-91) our interpretation of any graph will be limited by the ability of our measures to distinguish between different configurations, so we can only describe a graph through the number of metrics we invent to classify it. One hypothesis is that above a certain isovist coverage of a space any graph measures we take will become invariant (or vary only as a simple function of the set size). Determining such a characteristic limit to 'graph measure invariance' in specific cases would set a 'natural' upper limit on the number of locations that need to be used and it would also allow us to look at the effect of using, for example, distorted rather than regular grids. In addition, if it is possible to find such characteristic values for a system, then it would open up avenues to compare whole spatial environments with one another. As we have noted, average clustering coefficient and the characteristic path length (the average of 
the mean shortest path length for the whole system) have been used by Watts and Strogatz (1998) to analyse small worlds networks. By taking measures such as these for the whole system, we might hope to discover new characterisations for architectural types, and to classify their configurations.

\section{Conclusion}

In this paper we have presented a new approach to the application of isovists in spatial systems. Rather than investigate the properties of single isovists, as has been considered in the literature, we have constructed a graph by using isovist-generating locations as vertices, and visibility relationships between isovists as edges. The resulting graph is a visibility graph of locations within the space. By reinterpreting the set of isovists as a graph we have made the system more amenable to a new set of analytic tools, while retaining a mapping back to the original isovist interpretation through the neighbourhood size $k_{i}$ of a vertex. This allows us to discuss any measurement of the graph in terms of its spatial meaning. We have proposed two further measurements of the graph that may be useful for understanding the perception and use of architectural spaces. The measures are justified to some extent by the application of similar metrics to graphs of urban and building environments, and by their relevance to ongoing research; however, they are also intended as examples of the wider set of graph measures available. The clustering coefficient $C_{i}$ demonstrates a local measure of the graph, since it depends only on the relation between vertices in a neighbourhood, whereas the mean shortest path length $\bar{L}_{i}$ demonstrates a global measure of the graph, in that for each vertex it depends on the relative placement of all other vertices in the graph. We have shown results comparing the level of occupation of rooms found in a building with the results of an $\bar{L}_{i}$ analysis of that building and we have demonstrated that there is some quantitative correspondence between the two.

In architectural composition, a process of visualisation of space as being potentially occupied by groups of occupants and sequences of events is essential, though not necessarily conscious. Hill (1998, page 140) writes: "The architect and user both produce architecture, the former by design, the latter by inhabitation. As architecture is designed and experienced, the user has as creative a role as the architect." In this sense, sets of locations within the isovist of a point determine conditions of copresence of occupants and hence potential action and interaction. The isovists we employ are used to derive the graph of intervisible locations and hence the visibility graph is a tool with which we can begin consciously to explore the visibility and permeability relations in spatial systems. We must of course be careful to note that any population of a space will lead to changes in the visual field which we have not considered and that members of the population will experience a space through their personal memory of the previous spaces they themselves have moved through. However, by looking at relationships at both a local and a global level, we hope to capture the common experience of that space, and so visibility graph analysis may represent a step towards exploring the relationship between architects, as designers of spaces, and users, as architects of their own experience of space.

Acknowledgements. The authors would like to thank Dave Chapman and Fotini Kontou who helped with the early development of visibility graph analysis, the Space Syntax Laboratory, UCL, which helped with testing, and the anonymous referees for their comments and suggestions. The VR Centre for the Built Environment is funded by the Office of Science and Technology through a Foresight Challenge Award. David O'Sullivan is supported by an EPSRC Studentship held at the Bartlett Faculty of the Built Environment administered by the Centre for Advanced Spatial Analysis. 


\section{References}

Amidon E L, Elsner G H, 1968, "Delineating landscape view areas: a computer approach", Forest Research Note PSW-180, US Department of Agriculture, Washington, DC

Benedikt M L, 1979, “To take hold of space: isovists and isovist fields" Environment and Planning B $647-65$

Benedikt M L, Burnham C A, 1985, "Perceiving architectural space: from optic rays to isovists", in Persistence and Change Eds W H Warren, R E Shaw (Lawrence Erlbaum Associates, London)

Burrough P A, 1986 Principles of Geographical Information Systems for Land Resources Assessment (Clarendon Press, Oxford)

Conroy R, 2000 Spatial Navigation in Immersive Virtual Environments $\mathrm{PhD}$ thesis, Bartlett Faculty of the Built Environment, University College London, London

Curtis W J R, 1996 Modern Architecture Since 1900 (Phaidon, London)

Davis L S, Benedikt M L, 1979, "Computational models of space: isovists and isovist fields" Computer Graphics and Image Processing 11(3) 49-72

de Arruda Campos M B, 1997, "Strategic space: patterns of use in public squares of the city of London", in Proceedings of the First International Symposium on Space Syntax Space Syntax Laboratory, Bartlett School of Graduate Studies, University College London, London

deBerg M, Kreveld van M, Overmars M, Schwarzkopf O, 1997 Computational Geometry (Springer, Berlin)

De Floriani L, Marzano P, Puppo E, 1994, "Line-of-sight communication on terrain models" International Journal of Geographical Information Systems 8329 - 342

Fisher P F, 1991, "First experiments in viewshed uncertainty: the accuracy of the viewshed area" Photogrammetric Engineering and Remote Sensing 571321 - 1327

Fisher P F, 1995, "An exploration of probable viewsheds in landscape planning" Environment and Planning B: Planning and Design $22527-546$

Fisher P F, 1996, "Extending the applicability of viewsheds in landscape planning" Photogrammetric Engineering and Remote Sensing $621297-1302$

Futagawa Y, Neumeyer F, 1995 Mies van der Rohe: German Pavilion, International Exposition, Barcelona, Spain, 1928-29 (reconstructed 1986), Tugendhat House, Brno, Czecho, 1928-30 (ADA Edita, Tokyo)

Gallagher G L, 1972, "A computer topographic model for determining intervisibility", in The Mathematics of Large Scale Simulation Ed. P Brock (Simulation Councils, La Jolla, CA) pp $3-16$

Gibson J J, 1979 The Ecological Approach to Visual Perception (Houghton Mifflin, Boston, MA)

Giedion S, 1971 Architecture as the Phenomena of Transition (Harvard University Press, Cambridge, MA)

Hanson J, 1994, “'Deconstructing' architects' houses” Environment and Planning B: Planning and Design $21675-704$

Hanson J, 1999 Decoding Homes and Houses (Cambridge University Press, Cambridge)

Hill J, 1998 Occupying Architecture (Routledge, London)

Hillier B, Hanson J, 1984 The Social Logic of Space (Cambridge University Press, Cambridge)

Hillier B, Penn A, 1992, "Dense civilisations: the shape of cities in the 21st century" Applied Energy 43(1) $41-66$

Hillier B, Penn A, Hanson J, Grajewski T, Xu J, 1993, "Natural movement: or, configuration and attraction in urban pedestrian movement" Environment and Planning B: Planning and Design $2029-66$

Hillier B, Penn A, Dalton N, Chapman D, Redfern F, 1995, "Graphical knowledge interfaces: the extensive and intensive use of precedent databases in architecture and urban design", in Visual Databases in Architecture Eds A Koutamanis, H Timmermans, I Vermeulen (Avebury, Aldershot, Hants) pp 197-227

Hillier B, Major M D, Desyllas J, Karimi K, Campos B, Stonor T, 1996, “Tate Gallery, Millbank: a study of the existing layout and new masterplan proposal", technical report, Bartlett School of Graduate Studies, University College London, London

Huss R E, Pumar M A, 1997, "Effect of database errors on intervisibility estimation" Photogrammetric Engineering and Remote Sensing 63 415-424

Krüger M J T, 1979, "An approach to built-form connectivity at an urban scale: system description and its representation" Environment and Planning B 6 67-88

Lee J, Stucky D, 1998, "On applying viewshed analysis for determining least-cost paths on digital elevation models" International Journal of Geo-Information Science 12891 - 905 
Llobera M, 1996, "Exploring the topography of mind: GIS, social space and archaeology" Antiquity $70612-622$

Lynch K, 1976 Managing the Sense of Region (MIT Press, Cambridge, MA)

March L, Steadman P, 1971 The Geometry of Environment (Methuen, London)

Mills K, Fox G, Heimbach R, 1992, "Implementing an intervisibility analysis model on a parallel computing system" Computers and Geosciences 181047 - 1054

Ore O, 1963 Graphs and Their Uses (Random House, New York)

Peponis J, Wineman J, Rashid M, Hong Kim S, Bafna S, 1997, "On the description of shape and spatial configuration inside buildings: convex partitions and their local properties" Environment and Planning B: Planning and Design 24761 - 781

Steadman P, 1973, "Graph theoretic representation of architectural arrangement" Architectural Research and Teaching $2161-172$

Tandy C R V, 1967, “The isovist method of landscape survey”, in Symposium: Methods of Landscape Analysis Ed. H C Murray (Landscape Research Group, London) pages 9-10

van Fraassen B C, 1980 The Scientific Image (Clarendon Press, Oxford)

Wang J J, Robinson G J, White K, 1996, "A fast solution to local viewshed computation using grid-based digital elevation models" Photogrammetric Engineering and Remote Sensing 62 $1157-1164$

Watts D J, Strogatz S H, 1998, "Collective dynamics of 'small-world' networks" Nature 393 440 - 442

Wheatley D, 1995, "Cumulative viewshed analysis: a GIS based method of investigating intervisibility and its archaeological application", in Archaeology and GIS: A European Perspective Eds G Lock, Z Stancic (Taylor and Francis, London)

Wilson R J, 1996 Introduction to Graph Theory (Longman, Harlow, Essex)

Wilson R J, Beineke L W (Eds), 1979 Applications of Graph Theory (Academic Press, London) 
